\title{
Editorial
}

\section{Teaching Statistics: Announcing a new section of Model Assisted Statistics and Applications}

It is our great pleasure to announce a new section of the journal Model Assisted Statistics and Applications, "Teaching Statistics". Although its name is self-explanatory, it is worthwhile to discuss in more detail our vision of the section.

Most of you are aware that the 4th issue of the 2009 MASA volume constituted a special issue on teaching statistics. You are invited to visit the journal's web page and download the whole issue, because the IOS Press decided to make it free for all its readers to experience what the new section could cover. Nonetheless, treat what you will find there as only a sample of possible topics. What especially counts for us is the link between the statistical knowledge, teaching and applications. MASA's focus on applications will generally carry over to its teaching articles in the sense that we are more interested in creative reflections, practical strategies, and the scholarship of teaching than formally-structured educational research papers that are highly focused on theoretical frameworks and constructs. If you have interesting real-life data sets to offer, these can be found useful by many teachers and sometimes well-known data sets can be rediscovered. (Remember Cleveland's [1] discovery of a mysterious anomaly in the well-known barley data of Immer et al. [3]?) Simple-to-follow introductions to contemporary statistical methods, including those complex ones and quite specific for particular branches of science, are also encouraged (Johnson's work on the bootstrap [5], Eisenhauer's [2] on the regression through the origin, Peng and Ho's on logistic regression [7] are three good examples). Equally encouraged are software papers, describing efficient and non-trivial ways of analyzing data with particular methods in software packages, so that teachers could collect code for their class. I personally would be happy to see notes (usually short, but also longer) confronting contemporary statistical knowledge with common ways of analyzing a particular problem, examples including analysis of variance modeling for binomially distributed data confronted with generalized linear modeling [4], common misinterpretations of hypothesis testing [6], or applications of multiple comparison procedures, very common and yet considered inappropriate in many - if not all - contexts [8]. I find such works a great means for teachers of learning how they should revise their teaching to make it more up-to-date. Any teacher or student interested in statistics should find something interesting and thought-provoking here.

If you are not sure if your work (whether ready for submission or in scratch, or even just an idea that you've come up with yesterday) fits the section, don't hesitate to contact me and we'll discuss it. Don't bother about its length - both long and short papers are welcome, provided that the length is just right for the contents of the paper. This could be an original research or a discussion paper, entertaining or critical, presenting new research tools or refreshing old ones. What really counts for us is what the article is worth, and how it is written: don't be afraid of less formal style - it is fine to write in the first person and use active voice to engage the reader directly (e.g., writing "in my opinion" rather than "in the opinion of the author of the present paper", or "I discovered" rather than "it was discovered").

Ask yourself what you would like this section to cover and send us your feedback or simply submit! I will be more than happy to collect opinions from as many MASA readers as possible, so that they may inform our efforts to help and our journal and its new section to flourish. 
The platform for exchanging knowledge about teaching statistics that MASA is starting right now is of high priority for developing our teaching skills and statistical skills of our students. And we must not forget that statistics is one of those subjects that make most non-statistical and non-mathematical students sick. Ignoring that sad but all too true fact may be one of the reasons for failing in teaching. I myself never forget that while teaching, and the first thing I pass to my students is that statistics is not something to be afraid of, is not a means of scaring them and taking sleep from them: it's just a tool to discover and interpret phenomena of the world of Nature.

I don't know what it looks like in your country, but in Poland for faculties my colleagues and I teach for (agriculture, biology, food science, biotechnology etc.) we usually have 30 hours of lectures and the same amount of computer lab to teach a basic statistics course (which usually is the only course for undergraduate students), or sometimes even fewer. During that we should teach basic statistics, including population and sample; randomization; summary statistics; one-sample inference; two-sample inference; multi-sample inference (one-way analysis of variance and multiple comparisons, together with some aspects of experimental design); correlation; simple, multiple and polynomial regression; and basic categorical statistics. This is what I have to teach because that's included in the curriculum of Ministry of Science and Higher Education. Given students' knowledge about probability (poor) and statistics (none), 30 hours is not too many, is it? Yet another problem is that students in Poland do not in general possess sufficient knowledge of English to learn fully from English textbooks; most Polish textbooks for basic courses are not up-to-date for teaching contemporary statistics. Under such circumstances, teaching statistics is not an easy matter - especially when one wants to introduce some more advanced methodology such as generalized linear models.

Sorry for these digressions, but I decided to take this opportunity to share with you some of my experiences with teaching statistics; is it not what the section has been established for? Do share your thoughts, techniques, ideas, comments with us and other readers of the journal by submitting your notes and papers, and let our joint adventure begin!

I would like to express my thanks to the Editor-in-Chief, Dr Sarjinder Singh (Department of Mathematics, Texas A\&M University-Kingsville, USA), and the Co-Editor-in-Chief, Dr. Stan Lipovetsky (GfK Custom Research North America, USA), for appointing me to lead the Teaching Statistics Section, and to Drs. Larry Lesser (The University of Texas at El Paso, USA) and Arkady Shemyakin (University of St. Thomas, USA) for agreeing to join this section as Associate Editors.

We are looking forward to your feedback and let our joint adventure with the Teaching Statistics section of Model Assisted Statistics and Applications start!

\author{
Marcin Kozak \\ Department of Experimental Design and Bioinformatics \\ Warsaw University of Life Sciences - SGGW, Poland \\ Editor of the Teaching Statistics section, Model Assisted Statistics and Applications \\ E-mail: nyggus.masa@gmail.com \\ with contribution from \\ Lawrence M. Lesser \\ The University of Texas at El Paso, USA \\ Associate Editor of the Teaching Statistics section, Model Assisted Statistics and Applications
}

\title{
References
}

[1] W.S. Cleveland, Visualizing data, Summit, NJ: Hobart, USA, 1993

[2] J.G. Eisenhauer, Regression through the origin. Teaching Statistics 25 (2003), 76-80.

[3] F.R. Immer, H.K. Hayes and L.R. Powers, Statistical determination of barley varietal adaption. Journal of the American Society of Agronomy 26 (1934), 403-419.

[4] T.F. Jaeger, Categorical data analysis: Away from ANOVAs (transformation or not) and towards logit mixed models, Journal of Memory and Language 59 (2008), 434-446

[5] R.W. Johnson, An introduction to the bootstrap, Teaching Statistics 23 (2001), 49-54.

[6] M. Kozak, Correlation coefficient and the fallacy of statistical hypothesis testing, Current Science 95 (2008), 1121-1122. 
[7] C.J. Peng and T.H. Ho, Logistic regression analysis and reporting: A primer, Understanding Statistics 1 (2002), 31-70.

[8] R. Webster, Analysis of variance, inference, multiple comparisons and sampling effects in soil research, European Journal of Soil Science $\mathbf{5 8}$ (2007), 74-82. 